\title{
A importância dos serviços de atenção farmacêutica na formação e recuperação de saúde de pacientes diabéticos: uma revisão integrativa
}

The pharmaceutical care services importance in the education and health diabetic patients recovery: an integrative review

La importancia de los servicios de atención farmacéutica en la educación y la recuperación de la salud de los pacientes diabéticos: una revisión integradora

\section{Resumo}

Introdução: A Diabete Mellitus (DM) consiste em um distúrbio metabólico caracterizado por uma hiperglicemia persistente. Atualmente é uma epidemia global, acometendo mais de 424 milhões de indivíduos no mundo. Com isso, estruturação de um serviço de acompanhamento farmacêutico contribui para melhor adesão ao tratamento e redução de agravos oriundos do descontrole da patologia. Objetivo: Identificar através da revisão integrativa de literatura, qual o impacto das intervenções realizadas pelo farmacêutico dentro do serviço de atenção primária a saúde no controle dos parâmetros clínicos da diabete mellitus tipo II. Método: A construção do banco de dados foi realizada pelo Portal Regional da BVS. Foram incluídos estudos experimentais e não-experimentais, publicados entre os anos de 2015 e 2019. Após aplicação dos descritores, o total de artigos elegíveis encontrados foi de 188 textos integrais, em seguida os textos são revisados conforme adequação ao tema da pesquisa. Resultados: Ao todo, onze artigos preencheram os critérios de inclusão, sendo eles de caráter randomizados, estudos de coorte e revisões sistemáticas. Os artigos elencados buscaram mensurar o impacto das intervenções de monitoramento da DM realizadas pelo profissional farmacêutico, sua aplicabilidade e desfecho na melhoria clínica dos pacientes diabéticos. Para isso, são analisados os exames laboratoriais, verificação da pressão arterial e a presença de doenças correlacionadas, que influenciam na condição clínica primária do indivíduo. Conclusão: $O$ acompanhamento farmacêutico e as intervenções realizadas favorecem o controle dos parâmetros clínicos e laboratoriais, reduzindo o risco de complicações secundárias e descontrole da DM e doenças correlacionadas.

Palavras-chave: Diabete Mellitus; Atenção básica; Farmacêutico; Hiperglicemia.

\begin{abstract}
Introduction: A Diabetes Mellitus (DM) is a metabolic disorder characterized by persistent hyperglycemia. It is currently a global epidemic, affecting more than 424 million individuals worldwide. Thus, the structuring of a pharmaceutical follow-up service contributes to better adherence to treatment and reduction of injuries arising from the lack of control of the pathology. Objective: To identify, through an integrative literature review, the impact of interventions performed by the pharmacist within the primary health care service on the control of clinical parameters of type II diabetes mellitus. Method: The construction of the database was carried out by the BVS Regional Portal. Experimental and nonexperimental studies, published between 2015 and 2019, were included. After applying the descriptors, the total number of eligible articles found was 188 full texts, then the texts are proofreaders as appropriate to the research topic. Results: In all, eleven articles met the inclusion criteria, being them randomized, cohort studies and systematic reviews. The listed articles sought to measure the impact of interventions performed by the pharmacist, their applicability and outcome in the clinical improvement of diabetic patients. For this, laboratory tests, checking blood pressure and the presence of correlated diseases, which influence the primary clinical condition of the individual, are analyzed. Conclusion: The pharmaceutical follow-up and the interventions performed favor the control of clinical and laboratory parameters, reducing the risk of secondary complications and uncontrolled DM and related diseases.
\end{abstract}


Keywords: Diabetes mellitus; Basic care; Pharmaceutical; Hyperglycemia.

\section{Resumen}

Introducción: La Diabetes Mellitus (DM) es un trastorno metabólico caracterizado por una hiperglucemia persistente. Actualmente es una epidemia mundial que afecta a más de 424 millones de personas en todo el mundo. Así, la estructuración de un servicio de seguimiento farmacéutico contribuye a una mejor adherencia al tratamiento y a la reducción de las lesiones derivadas del descontrol de la patología. Objetivo: Identificar, mediante una revisión integradora de la literatura, cuál es el impacto de las intervenciones realizadas por el farmacéutico dentro del servicio de atención primaria de salud en el control de los parámetros clínicos de la diabetes mellitus tipo II. Método: La construcción de la base de datos fue realizada por el Portal Regional de la BVS. Se incluyeron estudios experimentales y no experimentales, publicados entre 2015 y 2019. Luego de aplicar los descriptores, el total de artículos elegibles encontrados fue de 188 textos completos, luego los textos son correctores de acuerdo a su adecuación al tema de investigación. Resultados: En total, once artículos cumplieron los criterios de inclusión, los cuales fueron estudios aleatorizados, de cohortes y revisiones sistemáticas. Los artículos enumerados buscaban medir el impacto de las intervenciones realizadas por el farmacéutico, su aplicabilidad y resultado en la mejoría clínica de los pacientes diabéticos. Para ello, se analizan las pruebas de laboratorio, el control de la presión arterial y la presencia de enfermedades correlacionadas, que influyen en la condición clínica primaria del individuo. Conclusión: El seguimiento farmacéutico y las intervenciones realizadas favorecen el control de los parámetros clínicos y de laboratorio, reduciendo el riesgo de complicaciones secundarias y DM no controlada y enfermedades relacionadas.

Palabras clave: Diabetes mellitus; Cuidados básicos; Farmacéutico; Hiperglucemia.

\section{Introdução}

A Diabetes Mellitus (DM) consiste em um distúrbio metabólico caracterizado por hiperglicemia persistente, decorrente de deficiência na produção de insulina e/ou na sua ação, a longo prazo, evolui para complicações crônicas micro e macro vasculares. Dentre outros aspectos, o envelhecimento da população, modificação socioeconômica, alimentação inadequada e a crescente incidência da obesidade e sedentarismo, são fatores determinantes para o aumento da prevalência da doença (Sociedade Brasileira do Diabete, 2017).

O diabetes é uma epidemia global, as estimativas indicam que 424 milhões de pessoas vivem com DM no mundo (8,3\%), podendo chegar a 626 milhões em 2045 (Sociedade Brasileira do Diabete, 2020). O Brasil ocupa o $4^{\circ}$ lugar no ranking dos países com maior taxa de incidência na doença, com 16 milhões de casos diagnosticados, atrás de China, Índia e Estados Unidos. Ainda de acordo com o estudo, a incidência cresceu $61,8 \%$ nos últimos dez anos, além disso, a patologia acomete cerca de 30\% de indivíduos com idade entre 19 a 64 anos em diferentes regiões do país (Sociedade Brasileira do Diabete, 2020). A doença, representa uma elevada carga financeira para a família e para os sistemas de saúde, decorrente de uma maior utilização dos serviços de saúde, perda de produtividade e dos cuidados requeridos para tratar suas complicações crônicas (Ministério da Saúde, 2017).

Dados informados pelo Vigitel (2018), no Brasil, o Estado com maior prevalência da doença é o Rio de Janeiro (10,4\%), seguido de Belo Horizonte (10,1\%) e São Paulo (10\%). O Estado do Pará registrou em 2016, 340 mil pessoas com o diagnóstico de diabetes, correspondendo a 6,3\% da população adulta do Estado, destes, cerca de $20 \%$ corresponde a jovens até 18 anos. No município de Belém, eram aproximadamente 68 mil indivíduos. Logo, a perspectiva para mudança desse cenário, prevê ações integradas da equipe multidisciplinar de saúde, incluindo o serviço farmacêutico, que visem a promoção, proteção e recuperação da saúde e prevenção de agravos de origem metabólica do diabete mellitus (Ramires, 2018).

Historicamente, o serviço farmacêutico era vinculado à descoberta e desenvolvimento de novos fármacos, novas metodologias e mecanização de indústrias farmacêuticas. A partir da década de 60, incorpora-se a filosofia de pharmaceutical care, marcando o movimento da farmácia clínica centrada no paciente (Brasil, 2015). Desde então, a participação do profissional redesenha o modelo de atenção às condições crônicas, como diabete mellitus, em nível de cuidados primários (Brasil, 2013). Em 2013, o Conselho Federal de Farmácia institui a Resolução № 585, que dispõe sobre a regulamentação das atribuições clínicas, dentre elas, o acompanhamento farmacoterapêutico, a conciliação terapêutica e a revisão da farmacoterapia (Brasil, 2014). 
O cuidado farmacêutico perpassa primariamente ao entendimento da dinâmica individual e familiar, com uma abordagem integral e individualizada, percebendo a família em seu espaço social, contexto socioeconômico e cultural (Brasil, 2015). O profissional avalia o contexto familiar, valorizando suas características individuais e coletivas. A prática deixa de ser centrada na doença e volta-se para o indivíduo, excluindo a visão médico-centrada, para formalizar o contexto equipe-pacientefamília (Lopes, Vilasbôas e Castellanos, 2017).

A estruturação de um serviço de acompanhamento farmacêutico no contexto de atenção primária à saúde, busca aumentar os níveis de adesão ao tratamento, favorecendo uma melhor eficácia terapêutica. O monitoramento de pacientes crônicos com DM contribui para a redução de agravos oriundos do descontrole da patologia, e as intervenções propostas, visam melhorar o controle dos parâmetros clínicos, com obtenção de resultados favoráveis hemoglibinaglicada (HbAc1) (Lopes, Vilasbôas e Castellanos, 2017).

Dessa forma, o objetivo do presente trabalho, por meio da revisão integrativa em literatura, busca identificar o impacto das intervenções realizadas pelo farmacêutico dentro do serviço de atenção primária à saúde no controle dos parâmetros clínicos da diabete mellitus tipo 2 .

\section{Método}

Trata-se de uma revisão integrativa, que inclui estudos experimentais e não-experimentais para compreensão de um fenômeno analisado, combinando conceitos, revisões, evidências e problemas metodológicos. Consolidando os resultados para posterior avaliação crítica dos achados na literatura.

Pesquisa de análise qualitativa e descritiva, para uma revisão integrativa, buscando evidenciar estudos científicos seguindo critérios determinantes como: síntese dos artigos; estabelecimento da questão da pesquisa; amostragem ou busca na literatura; categorização dos estudos; avaliação dos estudos incluídos na revisão; interpretação dos resultados; e por fim, síntese do conhecimento e apresentação da revisão (Santos, Pimenta e Nobre,2007).

Para formulação do problema, parte-se da seguinte pergunta norteadora: De que modo a implementação de um serviço de acompanhamento farmacêutico interfere na melhora dos parâmetros clínicos do portador de DM tipo 2? Para tal, utilizou-se do acrônimo PICO para se estabelecer os critérios de inclusão: População: Pacientes diagnósticos com diabete mellitus tipo II; Intervenção: acompanhamento farmacoterapêutico; Controle: Monitoramento dos índices glicêmicos; Desfecho: Melhora da adesão ao tratamento e dos parâmetros clínicos, com obtenção de resultados favoráveis de hemoglobina glicada (HbAc) e redução de agravos secundários.

A construção do banco de dados foi realizada por meio do Portal Regional da BVS (Biblioteca Virtual em Saúde), utilizando como estratégias de busca das informações, as bases de dados online indexados no National Library of Medicine (Pubmed), Literatura Latino-Americana de Ciências da Saúde (Lilacs) e Scientific Electronic Library Online (Scielo), e Medical Literature Analysis and Retrievel System Online (Medline). A estratégia de busca utilizou Descritores em Ciências da Saúde (DeCs) para seleção dos artigos, utilizando os domínios Atenção farmacêutica, Serviços de assistência farmacêutica, Diabete mellitus, Cuidado integral ao paciente e Consultório farmacêutico, vinculando os termos com os operadores booleanos "AND" e "OR". Os artigos selecionados foram incluídos sem restrição de idioma (português, inglês, islandês e espanhol), com publicação dentro dos últimos 5 anos.

Os critérios de inclusão foram revisões de literatura, meta-análises, dissertações e teses, que discorrem sobre a atenção farmacêutica voltada ao paciente diabético. A inclusão de artigos para a pesquisa ocorreu após leitura exploratória, aplicação dos descritores na plataforma BVS e principais bases de dados, triagem e elegibilidade dos artigos encontrados. 
A triagem dos artigos segue uma cronologia de inclusão, seleção de títulos coerentes e a leitura dos resumos, de forma independente, respeitando a pergunta chave da revisão. Inclui-se os artigos que respondam à questão da pesquisa com uma abordagem mínima após combinação de pelo menos dois descritores; com abordagem qualitativa adequada ao tema e a pergunta proposta; textos com publicação no período compreendido entre os anos de 2015 e 2020 e que foram encontrados na íntegra.

O total de artigos elegíveis encontrados foi de 188 textos integrais. Após a exclusão por duplicidade, restaram 120. Após aplicação de 3 ou 5 descritores, restaram 88. Inclusão de artigos publicados entre 2015 e 2020, 63. Artigos cujo tema se adequa a pesquisa, 57. Seguindo critérios de elegibilidade, com artigos encontrados na íntegra, 35. Incluídos para síntese após discussão, correspondem a 11 artigos conforme descrito na Figura 1.

Figura 1: Seleção dos artigos para revisão.

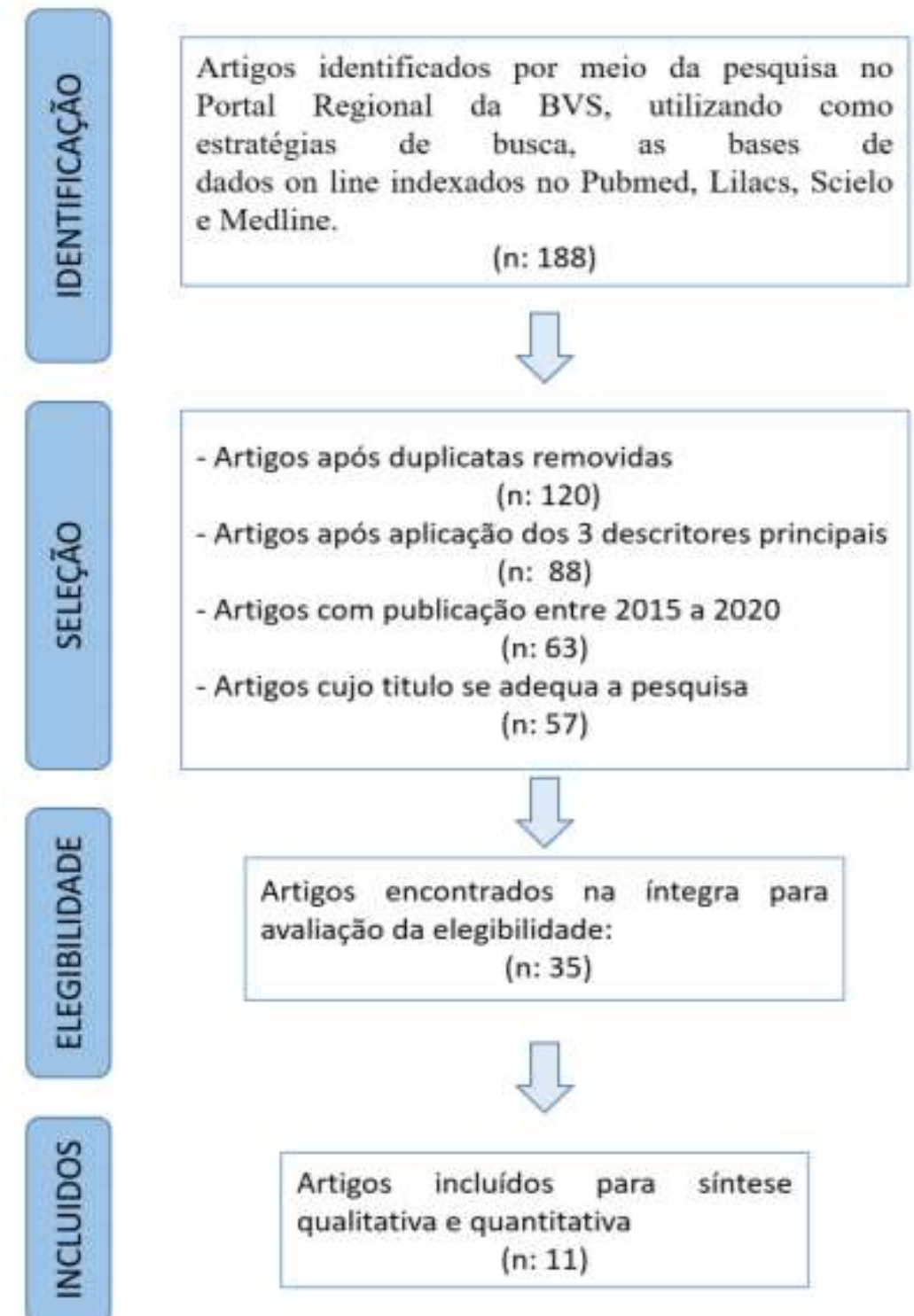

Fonte: Autores (2020).

\section{Resultados e Discussão}

Ao todo, onze artigos preencheram os critérios de inclusão. Os estudos incluídos foram publicados entre os anos de 2015 e 2019, sendo seis randomizados, dois estudos de coorte e três revisões sistemáticas. Os estudos foram realizados em 
diferentes países, o título dos artigos selecionados, a base de dados da publicação e as principais características dos artigos, como objetivo, tipo de estudo e conclusão dos autores, estão descritas na Tabela 1.

Na Tabela 2, consta o registro da síntese de evidências dos artigos elencados na tabela 1, considerando os desfechos clínicos, epidemiológicos, humanísticos e econômicos e quanto ao acesso a serviço. Korcegez, Sancar e Demirkan (2017), Siaw et al. (2017), Sharp et al. (2018) registram redução de recorrência, resistência/complicações, riscos, melhoria da segurança do paciente, eventos adversos, reações adversas, melhoria da satisfação do paciente, melhoria da qualidade de vida, do grau de informação sobre o medicamento e na melhoria ao acesso dos serviços farmacêuticos. Pereira et al. (2018) assinala redução de resistência/complicações, redução dos riscos e das reações adversas.

Gudlaugsdottir e Sigurdardottir (2018) mencionam: redução de recorrências, redução da resistência/complicações, melhoria da efetividade terapêutica, da segurança do paciente e melhoria do acesso aos serviços farmacêuticos. Lim et al. (2016), Chen et al. (2016) e Lyons et al. (2016), incluem além da redução de recorrências, de resistência/complicações, melhora da efetividade terapêutica, redução de riscos, melhoria da segurança do paciente, redução de eventos e reações adversas, melhora da satisfação do paciente, da qualidade de vida, do grau de informação sobre medicamentos e de acesso aos serviços farmacêuticos. Iqbal et al (2019), Jeong, Lee e Ji (2018) e Van Eikenhorst et al (2017) registram melhora da efetividade terapêutica, redução de riscos, melhoria da segurança, satisfação e qualidade de vida do paciente, assim como, melhora do grau de informação sobre medicamentos e de acesso aos Serviços farmacêuticos. 
Tabela 1: Perfil e características dos artigos selecionados.

\begin{tabular}{|c|c|c|c|c|}
\hline Artigo & Periódico & Objetivo & $\begin{array}{l}\text { Tipo de } \\
\text { estudo }\end{array}$ & Conclusão \\
\hline $\begin{array}{l}\text { A Review of Pharmacist-led } \\
\text { Interventions on Diabetes Outcomes: An } \\
\text { Observational Analysis to Explore } \\
\text { Diabetes Care Opportunities for } \\
\text { Pharmacists } \\
\quad \text { Autor: Iqbal et al. (2019). }\end{array}$ & Pubmed & $\begin{array}{l}\text { Avaliar as intervenções do } \\
\text { farmacêutico para pacientes para } \\
\text { melhorar o autogerenciamento com } \\
\text { diabetes mellitus e também para } \\
\text { melhorar os resultados clínicos do } \\
\text { diabetes mellitus. }\end{array}$ & $\begin{array}{l}\text { Revisão } \\
\text { sistemática } \\
\text { narrativa }\end{array}$ & $\begin{array}{l}\text { A intervenção liderada por farmacêuticos esteve } \\
\text { envolvida em todos os estudos incluídos na forma de } \\
\text { educação sobre diabetes e suas complicações, adesão } \\
\text { a medicamentos, estilo de vida e educação sobre } \\
\text { habilidades de autogestão, favorecendo a redução dos } \\
\text { níveis de hemoglobina glicada de } 0,75 \% \text {. }\end{array}$ \\
\hline $\begin{array}{l}\text { Study investigating the impact of } \\
\text { pharmacist involvement on the } \\
\text { outcomes of diabetes medication therapy } \\
\text { adherence program Malaysia. } \\
\text { Autor: Lim et al. (2016). }\end{array}$ & Pubmed & $\begin{array}{l}\text { Avaliar os resultados clínicos de } \\
\text { pacientes tratados por farmacêuticos } \\
\text { (DMTAC), em um hospital da } \\
\text { Malásia. Este foi um estudo } \\
\text { randomizado aberto. }\end{array}$ & $\begin{array}{l}\text { Randomizad } \\
\text { o prospectivo }\end{array}$ & $\begin{array}{l}\text { Os farmacêuticos gerenciados pela Clínica de Adesão } \\
\text { à Terapia de Medicamentos para Diabetes (DMTAC) } \\
\text { melhoraram significativamente o controle glicêmico e } \\
\text { o perfil lipídico de pacientes diabéticos. }\end{array}$ \\
\hline $\begin{array}{l}\text { Pharmaceutical care of elderly patients } \\
\text { with poorly controlled type } 2 \text { diabetes } \\
\text { mellitus: a randomized controlled trial } \\
\text { Autor: Chen et al. (2016). }\end{array}$ & Pubr & $\begin{array}{l}\text { Avaliar os efeitos do tratamento } \\
\text { farmacêutico no controle glicêmico } \\
\text { de pacientes idosos ambulatoriais } \\
\text { com diabetes tipo } 2 \text {. }\end{array}$ & $\begin{array}{c}\text { Randomizad } \\
\text { o }\end{array}$ & $\begin{array}{l}\text { O programa de intervenção farmacêutica forneceu } \\
\text { serviços que melhoraram o controle seguro a longo } \\
\text { prazo dos níveis de glicêmicos em pacientes idosos } \\
\text { ambulatoriais com diabetes e não aumentaram as } \\
\text { despesas médicas. }\end{array}$ \\
\hline $\begin{array}{l}\text { Pharmacist-Led } \\
\text { Interventions to Improve Diabetes } \\
\text { Outcomes. A Systematic Literature } \\
\text { Review and Meta-Analysis } \\
\text { Autor: Van eikenhorst et al. (2017). }\end{array}$ & & $\begin{array}{l}\text { Avaliar se as intervenções lideradas } \\
\text { por farmacêuticos para apoiar a } \\
\text { autogestão em pacientes com } \\
\text { diabetes melhoram os resultados } \\
\text { clínicos e relatados pelos pacientes. }\end{array}$ & $\begin{array}{l}\text { Revisão } \\
\text { sistemática e } \\
\text { meta analise }\end{array}$ & $\begin{array}{l}\text { As intervenções de autogestão melhoram } \\
\text { significativamente os valores de hemoglobina glicada } \\
\text { (HbA1c) em pacientes com diabetes. Esses } \\
\text { resultados sublinham o valor agregado dos } \\
\text { farmacêuticos no atendimento ao paciente, }\end{array}$ \\
\hline $\begin{array}{c}\text { Diabetes is a challenge: A ten year } \\
\text { follow up of people with diabetes } \\
\text { Autor: Gudlaugsdottir e } \\
\text { Sigurdardottir. (2018). }\end{array}$ & Medli & \begin{tabular}{lr|} 
Pesquisar & o resultado \\
do tratamento de parâmetros biológi \\
cos em pessoas com diabetes tipo 1 \\
e 2 em um atendimento primário e \\
comparar com \\
internacionais.
\end{tabular} & $\begin{array}{l}\text { Estudo de } \\
\text { coorte } \\
\text { retrospectivo }\end{array}$ & $\begin{array}{l}\text { Um controle mais rigoroso da glicose no sangue é } \\
\text { necessário para diminuir o risco de complicações, bem } \\
\text { como para reduzir os níveis de lipídios e pressão } \\
\text { arterial mais próximos das diretrizes internacionais. }\end{array}$ \\
\hline $\begin{array}{l}\text { Community Health Workers } \\
\text { Supporting Clinical Pharmacists in } \\
\text { Diabetes Management: A Randomized } \\
\text { Controlled Trial. } \\
\quad \text { Autor: Sharp. (2018). }\end{array}$ & Medline & $\begin{array}{l}\text { Avaliar a eficácia da atenção } \\
\text { farmacêutica e agentes comunitário } \\
\mathrm{s} \text { de saúde (ACS) na melhoria } \\
\text { do controle glicêmico em } \\
\text { uma população de minorias étnicas } \\
\text { de baixa renda. }\end{array}$ & Randomizado & $\begin{array}{l}\text { Não foram observadas diferenças significativas entre } \\
\text { a equipe do farmacêutico clínico- CHW e } \\
\text { o farmacêutico clínico sozinho na melhoria } \\
\text { do controle glicêmico em uma população }\end{array}$ \\
\hline
\end{tabular}




\begin{tabular}{|c|c|c|c|c|}
\hline $\begin{array}{l}\text { Avaliação da efetividade do } \\
\text { acompanhamento farmacoterapêutico } \\
\text { no controle do diabetes mellitus tipo } 2 \\
\text { em longo prazo } \\
\text { Autor: Pereira. (2018). }\end{array}$ & Lilacs & $\begin{array}{l}\text { Avaliar o desfecho clínico } \\
\text { de pacientes diagnosticados } \\
\text { com Diabetes mellitus tipo } 2 \text { (DM2) } \\
\text { após a alta de um serviço de AFT. }\end{array}$ & $\begin{array}{l}\text { Estudo de } \\
\text { coorte } \\
\text { retrospectivo }\end{array}$ & $\begin{array}{l}\text { Apesar das diferenças obtidas entre os grupos com o } \\
\text { AFT (Acompanhamento Farmacoterapêutico) não } \\
\text { permanecerem após quatro anos, os resultados clínicos } \\
\text { e laboratoriais não apresentaram piora significativa } \\
\text { nesse período }\end{array}$ \\
\hline $\begin{array}{l}\text { Impact of pharmacist-involved } \\
\text { collaborative care on the clinical, } \\
\text { humanistic and cost outcomes of high- } \\
\text { risk patients with type } 2 \text { diabetes } \\
\text { (IMPACT): a randomized controlled } \\
\text { trial. } \\
\quad \text { Autor: Siaw. (2017). }\end{array}$ & Medline & $\begin{array}{l}\text { Avaliar os resultados clínicos do } \\
\text { atendimento colaborativo } \\
\text { multidisciplinar versus } \\
\text { atendimento médico centrado } \\
\text { no diabetes, assim como, avaliar os } \\
\text { resultados humanísticos } \\
\text { econômicos entre os dois tipos de } \\
\text { atendimento. }\end{array}$ & $\begin{array}{l}\text { Randomizado } \\
\text { e prospectivo }\end{array}$ & $\begin{array}{l}\text { Os resultados clínicos, humanísticos e econômicos } \\
\text { positivos destacaram o valor do atendimento } \\
\text { colaborativo } \\
\text { para pacientes diabéticos asiáticos, multidisciplinar } \\
\text { a eficácia dessa abordagem no tratamento de doenças } \\
\text { crônicas. }\end{array}$ \\
\hline $\begin{array}{l}\text { Effect of a Pharmacist-Led Program on } \\
\text { Improving Outcomes in Patients with } \\
\text { Type } 2 \text { Diabetes Mellitus from Northern } \\
\text { Cyprus: A Randomized Controlled } \\
\text { Trial. } \\
\text { Autor: Korcegez, Sancar } \\
\text { e Demirkan. (2017). }\end{array}$ & Lilacs & $\begin{array}{l}\text { Avaliar o efeito do programa } \\
\text { de assistência farmacêutica no } \\
\text { controle glicêmico, determinado } \\
\text { pela hemoglobina glicada: Análise } \\
\text { da adesão a medicamentos e } \\
\text { autocuidado } \\
\text { pacientes diabéticos durante } 12 \\
\text { meses. }\end{array}$ & Randomizado & $\begin{array}{l}\text { A atuação do profissional farmacêutico em } \\
\text { um hospital público a nível ambulatorial foi associada } \\
\text { com melhorias significativas na redução da } \\
\text { hemoglobina glicada e outros desfechos secundários } \\
\text { em } 12 \text { meses randomizados controlados. }\end{array}$ \\
\hline $\begin{array}{l}\text { Effect of pharmaceutical care } \\
\text { interventions on glycemic control in } \\
\text { patients with diabetes: a systematic } \\
\text { review and meta-analysis } \\
\text { Autor: Jeong, Lee e Ji. (2018). }\end{array}$ & Pubmed & $\begin{array}{l}\text { Avaliar se o modelo de serviço de } \\
\text { atendimento farmacêutico pode } \\
\text { melhorar os resultados clínicos em } \\
\text { pacientes diabéticos. }\end{array}$ & $\begin{array}{l}\text { Revisão } \\
\text { sistemática }\end{array}$ & $\begin{array}{l}\text { O cuidado farmacêutico contínuo foi eficaz na } \\
\text { melhoria da hemoglobina glicada (HbA1c) em } \\
\text { pacientes com diabetes em LMIc e HIc. }\end{array}$ \\
\hline $\begin{array}{l}\text { The Medicines Advice Service } \\
\text { Evaluation (MASE): a randomised } \\
\text { controlled trial of a pharmacist-led } \\
\text { telephone based intervention designed } \\
\text { to improve medication adherence } \\
\text { Autor: Lyons et al. (2016). }\end{array}$ & Pubmed & $\begin{array}{l}\text { Testar a eficácia de um Serviço de } \\
\text { Aconselhamento Farmacêutico } \\
\text { (MASE) para melhorar a adesão dos } \\
\text { pacientes aos medicamentos } \\
\text { estabelecidos. }\end{array}$ & Randomizado & $\begin{array}{l}\text { As descobertas fornecem um suporte adicional ao } \\
\text { papel aprimorado dos farmacêuticos no apoio e } \\
\text { aconselhamento aos pacientes com seus } \\
\text { medicamentos para melhorar os resultados. }\end{array}$ \\
\hline
\end{tabular}

Fonte: Autores (2020). 
Tabela 2: Evidências de desfechos clínicos, epidemiológicos, humanísticos e econômicos e de acesso a serviços (1/2).

\begin{tabular}{|c|c|c|c|c|c|c|c|}
\hline \multirow[b]{2}{*}{ EVIDÊNCIA } & \multirow[b]{2}{*}{ DESFECHO } & \multicolumn{5}{|c|}{ AUTORES } & \multirow[b]{2}{*}{ Lyons et al. (2016). } \\
\hline & & $\begin{array}{l}\text { Korcegez, } \\
\text { Sancar e } \\
\text { Demirkan. } \\
(2017) .\end{array}$ & $\begin{array}{l}\text { Siaw et al. } \\
\text { (2017). }\end{array}$ & $\begin{array}{c}\text { Pereira } \\
\text { et al. } \\
(2018)\end{array}$ & $\begin{array}{c}\text { Gudlaugsdottir e } \\
\text { Sigurdardottir. } \\
\text { (2018). }\end{array}$ & Sharp et al. (2018). & \\
\hline \multirow{7}{*}{ Evidências Clínicas } & Redução de recorrências & $(*)$ & $(*)$ & $(*)$ & $(+)$ & $(+)$ & $(+)$ \\
\hline & $\begin{array}{lrr}\text { Redução de } & \text { resistência e/ou } \\
\text { complicações } & & \\
\end{array}$ & $(+)$ & $(+)$ & $(+)$ & $(+)$ & $(+)$ & $(+)$ \\
\hline & $\begin{array}{lll}\begin{array}{l}\text { Melhoria } \\
\text { terapêutica }\end{array} & \text { da } & \text { efetividade } \\
\end{array}$ & $(+)$ & $(+)$ & $(*)$ & $(+)$ & $(+)$ & $(+)$ \\
\hline & Redução de riscos & $(+)$ & $(+)$ & $(+)$ & $(*)$ & $(+)$ & $(+)$ \\
\hline & $\begin{array}{l}\begin{array}{l}\text { Melhoria da segurança do } \\
\text { paciente }\end{array} \\
\end{array}$ & $(+)$ & $(+)$ & $(*)$ & $(+)$ & $(+)$ & $(+)$ \\
\hline & Redução de eventos adversos & $(+)$ & $(+)$ & $(*)$ & $(*)$ & $(+)$ & $(+)$ \\
\hline & Redução de reações adversas & $(+)$ & $(+)$ & $(+)$ & $(*)$ & $(+)$ & $(+)$ \\
\hline \multirow{2}{*}{$\begin{array}{c}\text { Evidências } \\
\text { epidemiológicas }\end{array}$} & Redução de poli morbidade & $(*)$ & $(*)$ & $(*)$ & $(*)$ & $(*)$ & $(*)$ \\
\hline & Redução de mortalidade & $(*)$ & $(*)$ & $(*)$ & $(*)$ & $(*)$ & $(*)$ \\
\hline \multirow{3}{*}{$\begin{array}{l}\text { Evidências } \\
\text { humanísticas }\end{array}$} & $\begin{array}{l}\begin{array}{l}\text { Melhoria da satisfação do } \\
\text { paciente }\end{array} \\
\end{array}$ & $(+)$ & $(+)$ & $(*)$ & $(*)$ & $(+)$ & $(+)$ \\
\hline & Melhoria da qualidade de vida & $(+)$ & $(+)$ & $(*)$ & $(*)$ & $(+)$ & $(+)$ \\
\hline & $\begin{array}{l}\text { Melhoria do grau de informação } \\
\text { sobre medicamentos }\end{array}$ & $(+)$ & $(+)$ & $(*)$ & $(*)$ & $(+)$ & $(+)$ \\
\hline $\begin{array}{l}\text { Evidências } \\
\text { econômicas }\end{array}$ & Custo-benefício & $(*)$ & $(*)$ & $(*)$ & $(*)$ & $(*)$ & $(*)$ \\
\hline Acesso (outcomes) & $\begin{array}{l}\text { Melhoria do acesso aos Serviços } \\
\text { farmacêuticos }\end{array}$ & $(+)$ & $(+)$ & $(*)$ & $(+)$ & $(+)$ & $(+)$ \\
\hline
\end{tabular}

Legenda: $(+)=$ Positivo. $(*)=$ Não estudado. Nota: Devido o perfil dos artigos selecionados não foi possível classificar as evidências econômicas e epidemiológicas.

Fonte: Autores(2020). 
Research, Society and Development, v. 10, n. 13, e219101321149, 2021

(CC BY 4.0) | ISSN 2525-3409 | DOI: http://dx.doi.org/10.33448/rsd-v10i13.21149

Tabela 2: Evidências de desfechos clínicos, epidemiológicos, humanísticos, econômicos e de acesso a serviços (2/2).

\begin{tabular}{|c|c|c|c|c|c|c|}
\hline \multirow[b]{2}{*}{ EVIDÊNCIA } & \multirow[b]{2}{*}{ DESFECHO } & \multicolumn{5}{|c|}{ AUTORES } \\
\hline & & $\begin{array}{l}\text { Iqbal et al. } \\
\text { (2019). }\end{array}$ & $\begin{array}{l}\text { Lim et al. } \\
\text { (2016). }\end{array}$ & $\begin{array}{l}\text { Jeong, } \\
\text { Lee e Ji. } \\
(2018)\end{array}$ & $\begin{array}{c}\text { Van Eikenhorst et } \\
\text { al. (2017). }\end{array}$ & Chen et al. (2016). \\
\hline \multirow{6}{*}{ Evidências Clínicas } & Redução de recorrências & $(*)$ & $(+)$ & $(*)$ & $(*)$ & $(+)$ \\
\hline & $\begin{array}{lll}\begin{array}{l}\text { Melhoria } \\
\text { terapêutica }\end{array} & \text { da } & \text { efetividade } \\
\end{array}$ & $(+)$ & $(+)$ & $(+)$ & $(+)$ & $(+)$ \\
\hline & Redução de riscos & $(+)$ & $(+)$ & $(+)$ & $(+)$ & $(+)$ \\
\hline & Melhoria da segurança do paciente & $(+)$ & $(+)$ & $(+)$ & $(+)$ & $(+)$ \\
\hline & Redução de eventos adversos & $(*)$ & $(+)$ & $(*)$ & $(*)$ & $(+)$ \\
\hline & Redução de reações adversas & $(*)$ & $(+)$ & $(*)$ & $(*)$ & $(+)$ \\
\hline Evidências epidemiológica & Redução de poli morbidade & $(*)$ & $(*)$ & $(*)$ & $(*)$ & $(*)$ \\
\hline \multirow{3}{*}{ Evidências humanísticas } & Melhoria da satisfação do paciente & $(+)$ & $(+)$ & $(+)$ & $(+)$ & $(+)$ \\
\hline & Melhoria da qualidade de vida & $(+)$ & $(+)$ & $(+)$ & $(+)$ & $(+)$ \\
\hline & $\begin{array}{l}\text { Melhoria do grau de informação } \\
\text { sobre medicamentos }\end{array}$ & $(+)$ & $(+)$ & $(*)$ & $(*)$ & $(+)$ \\
\hline Evidências econômicas & Custo-benefício & $(*)$ & $(*)$ & $(*)$ & $(*)$ & $(*)$ \\
\hline Acesso (outcomes) & $\begin{array}{l}\text { Melhoria do acesso aos Serviços } \\
\text { farmacêuticos }\end{array}$ & $(+)$ & $(+)$ & $(*)$ & $(+)$ & $(+)$ \\
\hline
\end{tabular}

Legenda: $(+)$ = Positivo. $(*)$ = Não estudado. Nota: Devido o perfil dos artigos selecionados não foi possível classificar as evidências econômicas e epidemiológicas. Fonte: Autores (2020). 
São estudos de caráter randomizados Korcegez, Sancar e Demirkan (2017), Siaw et al. (2017), Sharp et al. (2018), Lim et al. (2016), Chen et al. (2016) e Lyons et al. (2016), cujo delineamento consiste na divisão de dois grupos, grupo controle (com atendimento habitual) e de intervenção (atendimento multidisciplinar colaborativo). Os estudos de Korcegez, Sancar e Demirkan (2017), Siaw et al. (2017) e Sharp et al. (2018) promovem intervenções de educação em saúde para controle dos parâmetros clínicos e laboratoriais. Os estudos Korcegez, Sancar e Demirkan (2017), Siaw et al. (2017), Sharp et al. (2018) e Lim et al. (2016) propuseram como modelo de análise das intervenções realizadas, a aplicação de questionários como o modelo de Morisky-Green, um método de verificação da adesão ao tratamento. Este teste consiste na análise de 4 perguntas, em que os pacientes são considerados aderentes à farmacoterapia se responderam "não" às quatro perguntas e não aderentes se responderem "sim" a qualquer pergunta.

O estudo de Korcegez, Sancar e Demirkan (2017) apresenta como método de trabalho, sessões presenciais de educação em saúde para realização da revisão da farmacoterapia, compartilhamento de conhecimentos acerca do processo saúde-doença do diabetes, estabelecimento de objetivos clínicos, ensinamento sobre o uso correto de medicamentos (incluindo métodos de administração de insulina) e atividades que promovam o autocuidado. Se necessário, realizar mudanças nos esquemas de medicação e encaminhamento médico. Como efeito do programa de assistência, observou-se melhores resultados clínicos e de adesão à farmacoterapia nos pacientes do grupo de intervenção em comparação com os resultados do grupo controle. O estudo de Siaw et al. (2017) ilustrou um impacto positivo do atendimento colaborativo na melhoria da glicemia, saúde mental e satisfação no controle do diabetes, apoiando a eficácia dessa abordagem no gerenciamento de outras doenças crônicas. A abordagem de Sharp et al. (2018) norteia os serviços de gerenciamento de medicamentos e doenças aos pacientes, seguindo um Protocolo de Gerenciamento Farmacêutico, que incluiu uma avaliação abrangente das necessidades, promoção da saúde, estabelecimento de metas centradas no paciente e educação, assim como, de intervenções para incentivar a mudança de comportamento.

O artigo de Chen et al. (2016) utilizou como vertente de estudo a educação em saúde, por meio de intervenções farmacêuticas para controle glicêmico de pacientes diabéticos com níveis de hemoglobina glicada descontrolada (HbA1c) de 9,0\%) durante 6 meses. Como resultado, observa-se uma diminuição dos níveis de hemoglobina glicada $(0,83 \%)$ após 6 meses de acompanhamento no grupo em que houve intervenção, em comparação com um aumento de 0,43\% no grupo controle.

A discussão expressa por Lyons et al. (2016) avalia como as intervenções melhoraram a adesão à medicação em pacientes com diabete não controlada por meio do Serviço de Aconselhamento sobre Medicamentos (MASE), com atendimento presencial e telefônico. O instrumento foi projetado para ser modificável para apoiar pacientes que tomam medicamentos prescritos para qualquer condição de longo prazo. O estudo também avaliou o grau de satisfação do usuário com o serviço de aconselhamento, tendo como resultado positivo $91,8 \%$.

Em seu estudo, Lim et al. (2016), ressalta que além do acompanhamento clínico de educação em saúde, verificou o nível de aderência pelo método de Morisky-Green. Este estudo foi realizado na Malásia, com inserção do profissional farmacêutico em um grupo de controle dos pacientes diabéticos, Serviço Clínico de Adesão à Terapia (DMTAC) para controle glicêmico, parâmetros lipídicos e adesão ao tratamento. O estudo aponta que houve mudança nos parâmetros clínicos e laboratoriais, o resultado de redução do perfil lipídico e de hemoglobina glicada foram mais significativos no grupo de intervenção do que o do grupo controle, corroborando os demais estudos.

Os artigos de Pereira et al. (2018) e Gudlaugsdottir e Sigurdardottir (2018) são estudos de coorte retrospectivo que analisou pacientes diabéticos durante um determinado espaço de tempo, apresentando parâmetros clínicos favoráveis, porém inconclusivos. O estudo de Gudlaugsdottir e Sigurdardottir (2018) demonstra que é preciso avaliar as métricas internacionais em conjunto com os aspectos psicossociais (fatores sociais, comportamentais, emocionais e ambientais complexos), para reduzir o 
viés da pesquisa. Já o de Pereira et al. (2018) indica em seu estudo que não houve diferença entre os grupos acompanhados pelo serviço de atenção farmacêutica, mas o grupo de intervenção manteve seus parâmetros clínicos dentro das métricas propostas.

Os estudos de Iqbal et al. (2019), Jeong, Lee e Ji (2018) e Van Eikenhorst et al. (2017) são revisões sistemáticas com avaliação por meta-análise. As propostas dos estudos se encaixam na temática abordada, voltada ao paciente diabético. As revisões avaliam a qualidade do tema e do quadro de intervenções, além de explanar sobre as perspectivas da temática dentro de diferentes cenários, possibilitando uma replicação eficiente. O presente estudo de Iqbal et al. (2019) avaliou 25 estudos, cujas intervenções foram realizadas pelo farmacêutico com atuação voltada para educação sobre modificação do estilo de vida, requisitos de exercícios, cuidados com os pés, gerenciamento de dieta adequada e cessação tabágica. A análise final do estudo, aponta uma melhora significativa nos níveis de hemoglobina glicada, com a redução média de $0,75 \%$. Os efeitos em outros resultados também foram significativos. A discussão exposta por Jeong, Lee e Ji (2018) analisou 37 estudos por metanálise, com foco na avaliação da diminuição dos valores de hemoglobina glicada no sangue, análise por 12 semanas. Neste estudo, observase uma associação entre os cuidados farmacêuticos e o gerenciamento clínico do diabetes. Apesar de identificar bons resultados em metanálise, a pesquisa aponta que o processo de intervenção só é eficaz quando ocorre por longos períodos, superior a 6 meses. A estratégia de intervenção maximiza a eficácia terapêutica e melhora o cuidado ao longo da vida em pacientes com diabetes.

Já o estudo de Van Eikenhorst et al. (2017), analisou 24 referências, revisando o efeito das intervenções de autogestão lideradas por farmacêuticos para pacientes com diabetes em resultados clínicos e em ensaios randomizados. Esta revisão demonstra que as intervenções realizadas pelo farmacêutico contribuem positivamente para o auto gerenciamento de pacientes diabéticos, com redução dos níveis de hemoglobina glicada em média de $0,71 \%$, favorecendo a redução do risco de complicações microvasculares.

\section{Limitações e viés}

Das limitações encontradas durante a pesquisa, conclui-se que estão relacionadas ao tamanho amostral e tempo de intervenção. Determinados estudos não apresentaram métodos claros e dados completos, dificultando a avaliação dos vieses da revisão, o entendimento da estruturação e replicação do serviço de acompanhamento. Pesquisas com tempo de intervenção reduzido, não exibiram resultados robustos. Entretanto, em todas as análises, observou-se resultados positivos no grupo de intervenção em comparação ao grupo controle, com redução dos níveis de hemoglobina glicada e melhoria das condições clínicas referentes ao paciente diabético.

\section{Conclusão}

Os artigos que promovem ações de educação em saúde são os de Korcegez, Sancar e Demirkan, (2017), Siaw et al. (2017), Sharp et al. (2018), Chen et al. (2016), Lyons et al. (2016), Iqbal et al. (2019), Jeong, Lee e Ji (2018) e Van Eikenhorst et al. (2017), que por meio de intervenções farmacêuticas, voltada para educação sobre modificação do estilo de vida, requisitos de exercícios, cuidados com os pés, gerenciamento de dieta adequada, cessação tabágica e outros, apresentaram melhores resultados durante o acompanhamento no grupo de intervenção em comparação ao grupo controle, exibindo uma redução significativa nos níveis de hemoglobina glicada descontrolada com acompanhamento superior a 6 meses.

O acompanhamento por períodos reduzidos, apesar de exibir um bom resultado, traz viés negativo para pesquisa, como apontado em Iqbal et al. (2019) e Jeong, Lee e Ji (2018). Além disso de acordo com Pereira et al. (2018) e Gudlaugsdottir e Sigurdardottir (2018), um bom índice de acompanhamento resulta de ações conjuntas de educação em saúde e avaliação dos aspectos psicossociais (fatores sociais, comportamentais, emocionais e ambientais complexos). As intervenções efetivas 
favorecem o controle dos parâmetros clínicos e laboratoriais, reduzindo o risco de complicações secundárias e descontrole de doenças correlacionadas.

Como possibilidade para novas pesquisas em relação ao acompanhamento farmacoterapêutico, sugerimos estudos comparativos em diversas regiões do país, para avaliação do impacto e da qualidade das intervenções realizadas no serviço de atenção primária à saúde para controle da diabete mellitus e suas complicações.

\section{Implicações práticas}

Recomenda-se a necessidade de estudos complementares quanto à regulamentação de padrões mínimos para a aplicação prática de simulação realística no contexto do processo ensino-aprendizagem. Seria oportuno, também, estudos econômicos para aferir a relação custo-eficácia e custo-benefício dos aplicativos disponíveis no mercado.

\section{Contribuições dos autores}

AJ concebeu a estratégia de pesquisa em conjunto com MA e MV. AJ fez as buscas bibliográficas nas bases de dados, a seleção dos artigos e a extração de dados sob a supervisão de MA e MV. Os autores leram e aprovaram a versão final do manuscrito. O conteúdo da revisão é de exclusiva responsabilidade individual dos autores.

\section{Declaração de conflito de interesses}

Os autores declaram que a pesquisa foi conduzida na ausência de quaisquer relações comerciais ou financeiras que possam ser interpretadas como um potencial conflito de interesse.

\section{Referências}

Brasil. (2015). Desenvolvimento da versão brasileira resumida do Diabetes Quality of Life Measure (DQOL-Brasil-8). Rev. bras. epidemiol., 18(4), 943-952.

Brasil. (2013). Resolução No 585 de 29 de agosto de 2013 do Conselho Federal de Farmácia. Dispões sobre a regulamenta as atribuições clínicas do farmacêutico e dá outras providências. https://www.cff.org.br/userfiles/file/resolucoes/585.pdf

Brasil. (2014). Ministério da Saúde. Serviços farmacêuticos na atenção básica à saúde / Ministério da Saúde, Secretaria de Ciência, Tecnologia e Insumos Estratégicos - Cuidado farmacêutico na atenção básica; caderno 1. Departamento de Assistência Farmacêutica e Insumos Estratégicos. - Brasília: Ministério da Saúde, 108 p.: il.

Chen, J., Ou, H. \& Lin, T. et al. (2016). Pharmaceutical care of elderly patients with poorly controlled type 2 diabetes mellitus: a randomized controlled trial. Int J Clin Pharm 38, 88-95. https://doi.org/10.1007/s11096-015-0210-4

Gudlaugsdottir, H. L. \& Sigurdardottir, A. K. (2018). Diabetes is a challenge: A ten year follow up of people with diabetes. Laeknabladid, 104(4), 171-176, 2018. https://pubmed.ncbi.nlm.nih.gov/29616657/.

Iqbal, M. Z., Khan, A. H., Iqbal, M. S. \& Syed Sulaiman, S.A. (2019). A Review of Pharmacist-led Interventions on Diabetes Outcomes: An Observational Analysis to Explore Diabetes Care Opportunities for Pharmacists.. J. Pharm Bioallied Sci. 11 (4),299-309. https://pubmed.ncbi.nlm.nih.gov/31619911/.

Jeong, S., Lee, M. \& Ji, E. (2018). Effect of pharmaceutical care interventions on glycemic control in patients with diabetes: a systematic review and metaanalysis, Ther Clin Risk Manag. 2018; 14: 1813-1829. https://doi.org/10.2147/TCRM.S169748.

Korcegez, E. I., Sancar, M. \& Demirkan, K. (2017). Effect of a Pharmacist-Led Program on Improving Outcomes in Patients with Type 2 Diabetes Mellitus from Northern Cyprus: A Randomized Controlled Trial. J Manag Care Spec Pharm, 23(5), 573-582. https://pubmed.ncbi.nlm.nih.gov/28448779/.

Lim, P. C. et al. (2016). Study investigating the impact of pharmacist involvement on the outcomes of diabetes medication therapy adherence program Malaysia. Pak J Pharm Sci. 29(2). https://pubmed.ncbi.nlm.nih.gov/27087103/.

Lopes, G. V. D. O., Vilasbôas, A. L. Q. \& Castellanos, M. E. P. (2017). Atenção Domiciliar na Estratégia Saúde da Família: avaliação do grau de implantação em Camaçari (BA). Saúde em Debate, 41, 241-254.

Lyons, I., Barber, N. \& Raynor, D. K. et al. (2016). The Medicines Advice Service Evaluation (MASE): a randomised controlled trial of a pharmacist-led telephone based intervention designed to improve medication adherence. BMJ Quality \& Safety. 25(10):759-69. https://pubmed.ncbi.nlm.nih.gov/26755665/.

Ministério da saúde. (2017). Agência Saúde: Obesidade. http://portalms.saude.gov.br/noticias/agencia-saude/28108-em-dez-anos-obesidade-cresce-60-nobrasil-e-colabora-para-maior-prevalencia-de-hipertensao-e-diabetes>. 
Research, Society and Development, v. 10, n. 13, e219101321149, 2021

(CC BY 4.0) | ISSN 2525-3409 | DOI: http://dx.doi.org/10.33448/rsd-v10i13.21149

Pereira, L. B et al. (2018). Avaliação da efetividade do acompanhamento farmacoterapêutico no controle do diabetes mellitus tipo 2 em longo prazo. Clinical \& Biomedical Research, 38(3). https://seer.ufrgs.br/hcpa/article/view/81089.

Ramires, E. K. N. M. et al. (2018). Prevalência e Fatores Associados com a Síndrome Metabólica na População Adulta Brasileira: Pesquisa Nacional de Saúde2013. Arq Bras Cardiol, 110(5), 455-466. https://www.scielo.br/j/abc/a/ZNrkLHkkRBhRctk9xJp5nHs/?lang=pt.

Santos, C. M. C., Pimenta, C. A. M. \& Nobre, M. R. C. (2007). A estratégia pico para a construção da pergunta de pesquisa e busca de evidências. Rev Latinoam Enfermagem, maio-junho; 15(3). https://www.scielo.br/j/rlae/a/CfKNnz8mvSqVjZ37Z77pFsy/?format=pdf\&lang=pt.

Sharp, L. K. et al. (2018). Community health workers supporting clinical pharmacists in diabetes management: a randomized controlled trial. Pharmacotherapy: The Journal of Human Pharmacology and Drug Therapy, 38(1), 58-68. https://pubmed.ncbi.nlm.nih.gov/29121408/.

Siaw, M. Y. L. et al. (2017). Impact of pharmacist-involved collaborative care on the clinical, humanistic and cost outcomes of high-risk patients with type 2 diabetes (IMPACT): a randomized controlled trial. Journal of clinical pharmacy and therapeutics, 42(4), 475-482.

Sociedade Brasileira de Diabetes. (2017). Conduta terapêutica no diabetes tipo 2: algoritmo SBD - Posicionamento Oficial SBD no 02/2017.

Sociedade Brasileira de Diabetes. (2020). Conduta terapêutica no diabetes tipo 2: algoritmo SBD - Diretrizes da sociedade brasileira do diabete 2019/2020. São Paulo.

Van eikenhorst, L., Taxis, K., Van dijk, L. \& De Gier, H. (2017). Pharmacist-Led Self-management Interventions to Improve Diabetes Outcomes. A Systematic Literature Review and Meta-Analysis. Front Pharmacol. 14; 8:891. https://pubmed.ncbi.nlm.nih.gov/29311916/.

Vigitel. (2018). Vigilância de fatores de risco e proteção para doenças crônicas por inquérito telefônico / Ministério da Saúde, Secretaria de Vigilância em Saúde, Departamento de Vigilância de Doenças e Agravos não Transmissíveis e Promoção da Saúde. Ministério da Saúde. 